\title{
Ovarian fibroma with elevated CA125 and OVA1 in postmenopausal woman
}

\author{
Farideh Keypour ${ }^{1 *}$, Ilana $\mathrm{Naghi}^{2}$, Saeed $\operatorname{Naghi}^{3}$ \\ ${ }^{1}$ Department of Obstetrics \& Gynecology, Iran University of Medical Sciences, Tehran, Iran \\ ${ }^{2}$ Department of Obstetrics \& Gynecology, Newark Beth Medical Center, New Jersey, USA \\ ${ }^{3}$ Student and data collector, Santa Monica College, Los Angeles, California, USA
}

Received: 13 November 2013

Accepted: 15 December 2013

\author{
*Correspondence: \\ Dr. Farideh Keypour, \\ E-mail: f-keypoor@sina.tums.ac.ir
}

(C) 2014 Keypour F et al. This is an open-access article distributed under the terms of the Creative Commons Attribution Non-Commercial License, which permits unrestricted non-commercial use, distribution, and reproduction in any medium, provided the original work is properly cited.

\begin{abstract}
Ovarian fibroma is a sexcord stromal tumor, which accounts for $4 \%$ of all ovarian tumors. It is uncommon benign tumor of ovary. Clinically, it is asymptomatic and may typically be detected during routine gynecological examinations. It may be associated with ascitis and hydrothorax known as Meig's Syndrome. Usually these ovarian tumors are unilateral, solid, hard masses with small areas of cystic degeneration. Due to their solid structure, these benign tumors are sometimes confused with malignant ovarian neoplasm during clinical evaluation. A 59 year old postmenopausal woman presented with flank pain and new onset urinary incontinence. Ultrasound evaluation revealed a pelvic mass. Labs showed elevated tumor marker level. The patient underwent total abdominal hysterectomy with bilateral salpingo-oophorectomy. Pathological examination however revealed an ovarian fibroma. During the postmenopausal years, the ovaries become atrophic. The incidence ovarian neoplasm increases with age. A suspicious or persistent complex mass and increased tumor marker like CA125 and OVA1 in post-menopausal woman requires surgical evaluation. The traditional diagnostic tools for ovarian tumors are bimanual examination, pelvic ultrasound and measuring serum biomarker like CA125 level, but these methods have low specificity and sensitivity. Now-a-days the role of some tumor marker such as OVA1in the management of ovarian tumor has been approved by FDA.
\end{abstract}

Keywords: Ovarian fibroma, Serum biomarker, CA125, OVA1

\section{INTRODUCTION}

Ovarian fibroma is derived from the connective tissue of ovarian cortex. This group of ovarian neoplasm is rare. Based upon data from the Surveillance Epidemiology and End Results (SEER) United States national cancer database from 1992 to 1999 , the overall incidence of this neoplasm was 0.2 per 100,000 women. We report a case of ovarian fibroma with elevated tumor marker like CA125 and OVA.

\section{CASE REPORT}

A 59-year-old woman (G4 P3 Ab1) presented with complaint of right flank pain, colicky in nature for ten months. She reported the new onset urinary frequency and incontinence. She had a history of spontaneous passage of right renal stone 4 years ago. She was in menopause since 9 years ago and never had postmenopausal vaginal bleeding.

Vaginal examination revealed atrophic uterus and a firm palpable mass in right lower quadrant (R.L.Q). Left ovary was unremarkable.

Transvaginal ultrasonography was suggestive of a right adnexal solid, multi septate mass measuring $80 \times 65 \times 70 \mathrm{~mm}$. The left adnexa and uterus were unremarkable. It was associated with mild ascites. 
Kidney ultrasonography revealed no nephrolithiasis and hydronephrosis.

Abdominal spiral CT scan with IV and oral contrast showed solid mass lesion $(80 \times 65 \times 70 \mathrm{~mm})$ in the right side of pelvic just superior to the uterus with some small cystic or necrotic changes, perilesional fat was intact. Adrenal glands, kidneys, pancreas, spleen were unremarkable, without enlarged lymph node.

Chest X-ray did not show any pleural effusion.

The patient's blood CA125 level was 140 U/ML (normal range $<30$ or $35 \mathrm{U} / \mathrm{ML}$ ).

$\begin{array}{ll}\mathrm{CEA}=4 / 2 & \text { Normal range }<5 \\ \mathrm{LDH}=420 & \text { Normal range }<480 \\ \mathrm{AFP}=3 / 5 & \text { Normal range }<5 / 8 \\ \mathrm{C} 19-9=2 / 7 & \text { Normal range }<5 \\ \beta-\mathrm{HCG}=8 / 2 & \text { Non pregnant }<10\end{array}$

The enlargement of the postmenopausal ovary immediate investigation was required. The patient underwent total abdominal hysterectomy with bilateral salpingooophorectomy. Exploration of the abdominal cavity did not yield any other abnormalities.

\section{Pathology repots}

1. Peritoneal fluid, cytology: Negative for malignant cell.

2. Omentum, biopsy: Unremarkable fibro fatty tissue.

3. Right ovary: Fibroma.

4. Left ovary: Unremarkable.

5. Endometrial: Weakly proliferative endometrial.

6. Cervix: Chronic cervicitis with nabothian cyst.

7. Fallopian tube: Unremarkable.

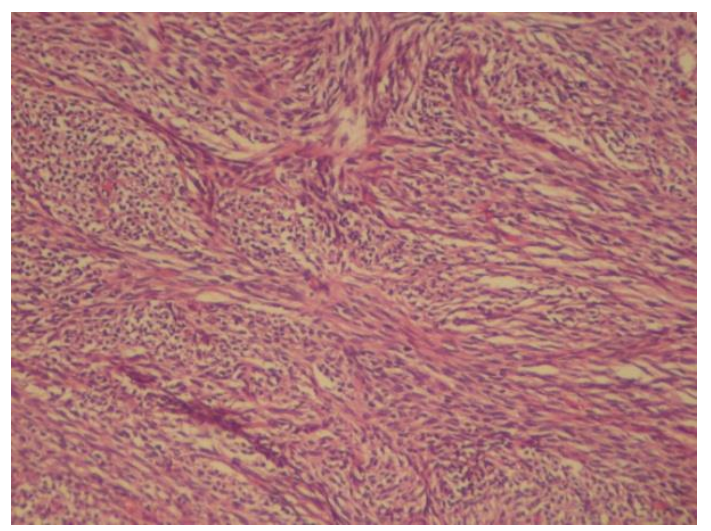

Figure 1: Microscopic finding in ovarian fibroma.

\section{DISCUSSION}

An ovarian fibroma is an ovarian mass of sex cord stromal origin. ${ }^{1}$ This ovarian mass is almost always benign in nature, frequency seen in middle-age women and not hormonally active. ${ }^{2}$
Symptoms of ovarian fibromas usually depend on their size, position in the pelvis or abdomen and rate of growth. Symptoms may include lower abdominal pain or pressure discomfort. ${ }^{3}$ Most of them are asymptomatic until they enlarge or involve adjacent organs and structures. ${ }^{3}$ Upon gross pathologic inspection, ovarian fibromas are firm and white or tan. They have a smooth lobulated surface. ${ }^{4}$

On microscopic examination, there are intersecting bundles of spindle cells producing collagen. ${ }^{4}$

Tumor markers are substances that are identified in higher than normal amounts in body secretion like blood, Urine, or body tissue of patients with specific malignancy. ${ }^{5}$ The tumor marker is produced by the tumor per se or as a response to the presence of cancer. It is used to fallow up of neoplasm tumor. The change in level generally correlates with response. ${ }^{5}$

Carcino Embryonic Antigen (CEA) levels are often elevated in patients with ovarian cancer, but the test is too nonspecific and insensitive to have much use in the management of ovarian neoplasm. ${ }^{5}$ The level of CA125, a surface glycoprotein associated mullerian epithelial tissue, is elevated in about $80 \%$ of patients epithelial ovarian cancers, particularly those with nonmucinous neoplasm. ${ }^{5-6}$

A test called OVA-1is meant to be used as tumor marker in ovarian cancer. ${ }^{5}$ The FDA (Food and Drug Administration) has approved this blood test called OVA1. ${ }^{5}$ The OVA1panel measures the level of five proteins in the blood. These biomarkers include: transthyretin, beta 2-microglobulin, apolipoprotein a1, CA125 cancer antigen and transferrin. The results of these lab tests converts an algorithm which helps indicate whether the ovarian mass is malignant. ${ }^{5} \mathrm{HE}-4$ is another ovarian tumor marker can be used like CA125 to guide treatment. Some ovarian cancers have too much of a gene called HE-4, and make more protein called HE-4.Level of this protein can be measured in the blood. ${ }^{5}$ On ultrasound, fibroma most commonly manifest as solid, hypo-echo mass pattern, calcification may be identified. ${ }^{7}$

CT scan: fibroma usually unilateral manifest as diffuse, slightly hypo attenuating mass with poor, very contrast enhancement. $^{3,6,7}$

MRI: signal characteristic include homogeneous, relatively low intensity. When containing scattered highsignal- intensity areas representing cystic degeneration. ${ }^{7}$ The main differential diagnosis is pedunculated subserosal uterine leiomyoma. ${ }^{2}$ Surgical removal ovarian fibroma is recommended because of the low potential of malignancy. ${ }^{2}$

Result: Definitive diagnosis of ovarian mass in postmenopausal woman requires surgery; however, many procedures performed for a pelvic mass ultimately a 
benign adnexal mass. Use of tumor marker with other finding in ultrasound, CT scan and MRI in woman who have an adnexal mass can help to diagnose her disease. Clinical findings suggesting benign adnexal mass are: unilateral, cystic, mobile, smooth, no ascites, slow patient. ${ }^{6}$ It is important to measure CA125 before surgery so that it can be used as a baseline value to monitor the success of treatment if ovarian cancer is found. ${ }^{5}$ The OVA-1 marker test should not be used for screening of ovarian cancer but may be used to determine the need for surgery in patient presenting with an ovarian mass. ${ }^{5}$

Conclusion: Enlargement of the postmenopausal ovary requires immediate investigation. ${ }^{6}$ All women at high risk for ovarian cancer must be done screening test: Factors that increase their risk of ovarian cancer include the following:

- Having abnormalities in a gene, called BRCA1 or BRCA2

- The lifetime risk of ovarian cancer is $54 \%$ for BRCA1 and $23 \%$ for BRCA2 mutation. The risk of breast cancer in women with a BRCA1 or BRCA2 mutation may be as high as $56 \%$ to $87 \%$.

- Having genes that are linked to hereditary nonpolyposis colorectal cancer, breast, endometrial, and ovary (also called Lynch syndrome II or HNPCC, This syndrome, which includes multiple adenocarcinomas).

- Women with older age

- Having one or more relatives with breast or ovarian cancer

- Gravity=0

- Obesity

Screening includes a combination of biomarker test like CA125, OVA1 panel and pelvic imaging. Some experts recommend this combination of tests every six months, beginning at age 35 or 5 to 10 years before the youngest relative was diagnosed with breast or ovarian cancer. ${ }^{6}$

The need for providing doorstep services to people in rural India and training rural health workers to motivate every pregnant woman to seek expert care during her pregnancy cannot be overemphasized and must be undertaken with utmost priority.

Funding: No funding sources

Conflict of interest: None declared

Ethical approval: Not required

\section{REFERENCES}

1. Berek \& Hacker's. Cancer chemotherapy. In: Jonathan S. Berek, Neville F. Hacker, eds. Berek \& Hacker's Gynecologic Oncology. 5th ed. Philadelphia: Lippincott Williams \& Wilkings; 2010: 195-196.

2. Philip J., Disaia, William T., Cancers of the female reproductive tract. In: Creasman, eds. Clinical Gynecologic Oncology. 7th ed. UK: British Library, Mosby Elsevier; 2007: 294-238.

3. Sheila Jorge Adad, Valeria Limb Laterza, Carlos David Teixeira dos Santos et al. "Cellular Fibroma of the Ovary with Multiloculated Macroscopic Characteristics: Case Report". Case Reports in Medicine. 2012;2012:5.

4. Foundations in Diagnostic Pathology. In: Nucci, Marisa R, Oliva, Ester, Goldblum, John R., eds. Gynecologic Pathology. 1st ed. New York: Elsevier Churchill Livingstone; 2009: 446-448.

5. Frederick Rand Ueland, Andrew John Li. Serum biomarkers for evaluation of an adnexal mass for epithelial carcinoma of the ovary, fallopian tube, or peritoneum, 2013. Available at: URL: http//:www.Wolters Kluwer health/ up to date medicine. Accessed 17 April 2013.

6. Berek \& Novak's Gynecology. In: Berek Jonathan S., Berek Deborah L., eds. Berek \& Novak's Gynecology. 15th ed. Philadelphia, PA: Lippincott Williams and Wilkins; 2012: 1264.

7. Callen PW. Ultrasonography in Obstetrics and Gynecology. 5th ed. Philadelphia, PA: Saunders Elsevier; 2008: 1069-1978.

DOI: $10.5455 / 2320-1770 . i j r \operatorname{cog} 20140348$

Cite this article as: Keypour F, Naghi I, Naghi S. Ovarian fibroma with elevated CA125 and OVA1 in postmenopausal woman. Int J Reprod Contracept Obstet Gynecol 2014;3:236-8. 